\title{
Picophotonics: Visible invisible
}

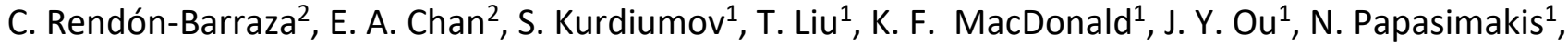
T. Pu ${ }^{1}$, G. Yuan ${ }^{2}$ and N. I. Zheludev ${ }^{1,2}$

1. Optoelectronics Research Centre and Centre for Photonic Metamaterials, University of Southampton, Highfield, Southampton, SO17 1BJ, UK

2. Centre for Disruptive Photonic Technologies, School of Physical and Mathematical Sciences and The Photonics Institute, Nanyang Technological University Singapore, 637378, Singapore

We report on recent advances in applications of deep learning and topologically structured light to farfield non-destructive imaging with deep subwavelength resolution and picometric metrology. 